

\title{
Keywords
}

bisexual; gay; identity; HIV; condom use; substance use before sex

It is well-documented that gay and bisexual men are disproportionately affected by HIV. In 2015, $82 \%$ of diagnosed HIV infections among adolescent and adult men were attributed to men who have sex with men (MSM) (CDC, 2016) and young MSM are the demographic group in which rates are increasing most (CDC, 2015). However, because transmission categories are based on behavior in domestic HIV surveillance systems, few data are available for self-identified gay versus bisexual men. Most research on MSM relies on

Corresponding author: Brian A. Feinstein, Northwestern Institute for Sexual and Gender Minority Health and Wellbeing, 625 N. Michigan Ave. \#14-047, Chicago, IL 60611, brian.feinstein@ northwestern.edu, 312-503-6516. 
samples of primarily gay men and, when bisexual men are considered, it is typically as an extension of gay men (e.g., "gay and bisexual men") with little-to-no distinction. There has been some attention to behaviorally bisexual men (i.e., men who have sex with men and women, MSMW), but scholars have criticized behavioral categories for obscuring important differences across identities, which are critical to understanding and eliminating health disparities (Bauer \& Brennan, 2013; Young \& Meyer, 2005). Further, even behavioral transmission categories used in domestic HIV surveillance systems have historically aggregated MSM and MSMW into a single category (Friedman et al., 2014). In order for HIV prevention interventions to be effective for both self-identified gay and bisexual young men, it is first necessary to understand the extent to which each group engages in HIV risk behaviors. To address this, the current study examined differences in HIV risk behaviors between self-identified gay and bisexual young men who are HIV-negative, and differences in HIV risk behaviors with male versus female partners among the bisexual men.

\section{Condomless sex}

Most early research on bisexual men's risk for HIV focused on behaviorally bisexual adult men (see Doll \& Beeker, 1996). These studies generally found that behaviorally bisexual adult men were less likely to report condomless anal sex (CAS) compared to behaviorally homosexual adult men, but this was due, in part, to behaviorally bisexual adult men being less likely to report any anal sex regardless of condom use (Stokes, Vanable, \& McKirnan, 1997). As noted, behavioral transmission categories can mask important differences related to self-identified sexual orientation (Bauer \& Brennan, 2013; Young \& Meyer, 2005). The use of behavioral transmission categories was intended to reflect the notion that behaviors rather than identities contribute to HIV transmission. However, behaviors occur in the context of the social and cultural norms and values of communities (Young \& Meyer, 2005).

In fact, previous research has demonstrated that peers and community members play important roles in shaping sexual norms and behavior (McKechnie, Bavinton, \& Zablotska, 2013). Given that bisexual individuals report less connection to the broader sexual minority community compared to gay/lesbian individuals (Balsam \& Mohr, 2007; Cox, Vanden Berghe, Dewaele, \& Vincke, 2010), it is likely that their sexual behavior is influenced by different social and cultural norms and values. In line with this possibility, Lever and colleagues (1992) identified several differences between self-identified bisexual men and behaviorally bisexual men who did not self-identify as bisexual (Lever, Kanouse, Rogers, Carson, \& Hertz, 1992). Specifically, they found that self-identified bisexual men were less likely to be married, less likely to have children, more likely to have ever had anal sex, and more likely to describe their sexual behavior as "predominately homosexual." These findings suggest that self-identified bisexual men are embedded in different social communities and have different patterns of sexual behavior than behaviorally bisexual men who do not self-identify as bisexual. As such, it is important to consider self-identified sexual orientation in order to understand differences in HIV risk behavior between gay and bisexual men.

Over time, there has been increased attention to self-identified bisexual men, but studies examining differences in HIV risk behaviors between self-identified gay and bisexual men 
have led to mixed findings. There is some evidence that condomless sex does not differ between self-identified gay and bisexual adolescent men (Everett, Schnarrs, Rosario, Garofalo, \& Mustanski, 2014), but there is also evidence that it depends on partner and relationship characteristics. For example, two studies found that self-identified bisexual young men were less likely to report CAS with main/serious male partners compared to selfidentified gay young men (Agronick et al., 2004; Lachowsky et al., 2015), but one of these studies also found that self-identified bisexual young men were more likely to report insertive CAS with non-main male partners compared to self-identified gay young men (Agronick et al., 2004). Further, in a sample of HIV-positive adults, self-identified bisexual men were less likely to report CAS with male partners compared to self-identified gay men (Doll et al., 1992). In contrast, self-identified bisexual men were more likely to report CAS with female partners compared to self-identified gay men, but only one self-identified gay man reported CAS with a female partner.

One potential reason for these mixed findings is that studies have used various methodological approaches for assessing sexual behavior, such as focusing on a single partnership (e.g., one's last sexual encounter) and aggregating across multiple partnerships. These approaches assume that a given individual's condom use is consistent across partnerships, but research has demonstrated that individuals vary in their use of condoms with different partners (Cooper, 2010; Mustanski, Newcomb, \& Clerkin, 2011; Newcomb \& Mustanski, 2013). For this reason, researchers have recommended using partner-level data (i.e., data on a given individual's behavior across multiple partnerships) to examine HIV risk at the within-person level (Mustanski et al., 2011). This approach allows each participant to serve as their own control by comparing their behavior with one partner to their behavior with another partner. Therefore, the current study used partner-level data to examine differences in HIV risk behaviors between self-identified gay and bisexual young men.

Despite considerable interest in bisexual men as a potential bridge for HIV transmission from male to female partners (Jeffries, 2014), few studies have examined differences in bisexual men's condom use with male versus female partners. In qualitative studies, behaviorally bisexual adult men report unique barriers to condom use with both male and female partners (e.g., men are less insistent about the need to use condoms and women are perceived as posing minimal risk for HIV) (Dodge, Jeffries, \& Sandfort, 2008; Hubach et al., 2014; Munoz-Laboy \& Dodge, 2007). As such, it is not surprising that quantitative studies have led to mixed findings. For example, one study found that behaviorally bisexual adult men were less likely to use condoms with male partners compared to female partners (Jeffries \& Dodge, 2007), but another study found that partner gender was not associated with condom use among young MSM (Mustanski et al., 2011). To our knowledge, only one previous study has examined differences in condom use with male versus female partners in a sample of self-identified bisexual men. Stokes, McKirnan, and Burzette (1993) found that self-identified bisexual adult men were less likely to report consistent condom use with female partners compared to male partners. Further, condom use was similar across steady and casual partners with the exception that condom use during vaginal intercourse was more frequent with casual partners than with steady partners. However, because they did not use partner-level data, it remains unclear if a given self-identified bisexual man's condom use differs with a male partner versus a female partner. 


\section{Substance use before sex}

Substance use is a risk factor for condomless sex (Drumright, Patterson, \& Strathdee, 2006; Mustanski, Newcomb, Du Bois, Garcia, \& Grov, 2011; Vosburgh, Mansergh, Sullivan, \& Purcell, 2012), and gay and bisexual youth are more likely to use substances before sex compared to heterosexual youth (Herrick, Marshal, Smith, Sucato, \& Stall, 2011). However, few studies have examined differences in substance use before sex between self-identified gay and bisexual men, and findings to date have been mixed. For example, one study found that self-identified bisexual young men were more likely to use substances before sex compared to self-identified gay young men (Agronick et al., 2004), but this was not replicated in a sample of adolescents (Everett et al., 2014). Given that bisexual individuals face discrimination from both heterosexual and gay/lesbian individuals (see Feinstein \& Dyar, 2017), self-identified bisexual men may be more likely to use substances before sex compared to self-identified gay men, because they may rely on substances to cope with enacted and internalized stigma. If self-identified bisexual men use substances before sex to cope with stigma, then they may experience reduced inhibitions and decision-making capabilities, contributing to reduced condom use. Additionally, if self-identified bisexual men have internalized negative societal attitudes toward bisexuality, then they may not feel comfortable talking to their partners about sex and sexual health. Further, they may not feel empowered to negotiate condom use with their partners, especially if they are under the influence of substances.

Finally, to our knowledge, no previous studies have examined the association between partner gender and substance use before sex among self-identified bisexual men. However, previous research on gender differences in substance use and previous research on bisexual men's experiences with partners of different genders can offer insight into this association. First, there are well-documented gender differences in substance use and disorders, both of which are more common among men than women (Carliner et al., 2017; Delker, Brown, \& Hasin, 2016; Grant et al., 2004; Kessler et al., 2005), and this extends to alcohol use before sex (Thompson, Eaton, Hu, Grant, \& Hasin, 2014). Further, substance use is common among young MSM (Newcomb, Ryan, Greene, Garofalo, \& Mustanski, 2014). There is also some evidence that behaviorally bisexual men are more likely to use alcohol with male sexual partners compared to female sexual partners (Martinez et al., 2012). In qualitative studies, behaviorally bisexual adult men, as well as non-gay-identifying adult MSM (i.e., those who identify as bisexual, heterosexual, or another non-gay identity), describe using alcohol with male sexual partners to feel more comfortable having sex with other men (Harawa et al., 2008; Martinez et al., 2012). As such, self-identified bisexual men may be more likely to use substances before sex with male partners compared to female partners.

However, there is also evidence to support the opposite possibility (i.e., that self-identified bisexual men may be more likely to use substances before sex with female partners compared to male partners). Previous research has demonstrated that behaviorally bisexual adult men perceive female partners as posing less risk for HIV compared to male partners (Dodge et al., 2008; Hubach et al., 2014). This has been described as a barrier to bisexual men using condoms with female partners, but it is also possible that this contributes to bisexual men being less concerned about the disinhibiting effects of substance use before sex 
with female partners. As such, self-identified bisexual men may be more likely to use substances before sex with female partners compared to male partners. Another plausible explanation for this possibility is that behaviorally bisexual adult men perceive female partners as being less accepting of their bisexual orientation compared to male partners (Dodge et al., 2008). Therefore, self-identified bisexual men may be less comfortable during sex with female partners (e.g., due to concerns about acceptance), in which case they may be more likely to rely on the disinhibiting effects of substance use before sex with female partners. The extent to which self-identified bisexual men use substances before sex with partners of different genders may also depend on whether the partner is serious or casual, given that single adults are more likely to regularly drink alcohol before sex than partnered adults (Thompson et al., 2014). In sum, few studies have compared self-identified gay and bisexual men on substance use before sex, and it remains unclear if self-identified bisexual men are more or less likely to use substances before sex with different types of partners.

Of note, it is particularly important to understand the extent to which self-identified gay and bisexual men engage in HIV risk behaviors during adolescence and emerging adulthood. As noted, rates of HIV are increasing the most among young MSM (CDC, 2015), and there are a lack of evidence-based HIV prevention interventions for young MSM, especially bisexual men (Hergenrather, Emmanuel, Durant, \& Rhodes, 2016). Adolescence and emerging adulthood are critical developmental periods to shape sexual health among gay and bisexual men. On average, gay and bisexual men first become aware of their same-sex attraction before adolescence and first self-identify as gay/bisexual during adolescence (Dunlap, 2016; Martos, Nezhad, \& Meyer, 2015). Further, adolescence and emerging adulthood are periods of profound change (Arnett, 2000; Jessor, 1992; Mustanski, Kuper, \& Greene, 2014), when perceived "benefits" of engaging in risk behavior can outweigh knowledge of "risks" (Parsons, Siegel, \& Cousins, 1997; Siegel, Cuccaro, Parsons, Wall, \& Weinberg, 1993). Therefore, understanding risk behavior among young gay/bisexual men has the potential to inform developmentally-appropriate HIV prevention.

\section{The current study}

In sum, little is known about HIV risk behavior among self-identified bisexual men, and several important questions remain. To fill these gaps, we used four waves of data spanning 24-months from a cohort of young MSM ages 16-29. At each wave (six-months apart), participants reported on their behavior with up to four partners during the prior six months. Therefore, we were able to examine within-person associations between partner/relationship characteristics and HIV risk behaviors. First, in the full sample, we examined: (1) predictors of condomless sex, including sexual orientation (gay versus bisexual), relationship type (serious versus casual), substance use before sex (alcohol and marijuana use), and the interactions between sexual orientation and each of the other predictors; and (2) predictors of substance use before sex, including sexual orientation, relationship type, and their interaction. Then, in the subsample of self-identified bisexual men, we examined: (3) predictors of condomless sex, including partner gender (male versus female), relationship type, substance use before sex, and the interactions between partner gender and each of the other predictors; and (4) predictors of substance use before sex, including partner gender, relationship type, and their interaction. Of note, for brevity, we refer to participants as "gay 
and bisexual men" instead of "self-identified gay and bisexual men" throughout the methods and results.

\section{Methods}

\section{Participants and Procedures}

Data were collected between February 2015 and August 2017 as part of the RADAR study -an ongoing longitudinal cohort study of young MSM in the Chicago metropolitan area (Janulis et al., 2017; Mustanski, Swann, Newcomb, \& Prachand, 2017). The broad goal of the RADAR study is to understand multilevel influences on HIV and substance use among young MSM. We utilized multiple recruitment methods to achieve a multiple cohort, accelerated longitudinal design (Duncan, Duncan, \& Hops, 1996). First, a subset of participants from two cohorts of young MSM-Project Q2 (first recruited in 2007) and Crew 450 (first recruited in 2010)—were eligible for enrollment. In 2015, a third cohort of young MSM was recruited. Cohort members were required to meet the following criteria: 16-29 years old, assigned male at birth, English-speaking, and either had sex with a man in the past year or identified with a sexual minority label (e.g., gay, bisexual). We expanded the RADAR cohort by allowing cohort members to recruit their serious partners at each visit, thereby creating a dynamic dyadic network. All serious partners who were assigned male at birth and ages 16-29 were eligible for enrollment into the cohort. Serious partners who were assigned female at birth or older than 29 were eligible to complete a one-time visit, but they were not eligible to be enrolled into the cohort. Lastly, cohort members were allowed to refer up to three peers for enrollment into the cohort (who also had to meet the inclusion criteria).

Participants complete study visits every six months. At each visit, participants complete a psychosocial survey, a network interview, and biomedical specimen collection for testing (HIV, STI, and/or drug screening) and banking. Survey data are collected using a computerassisted self-interview (CASI). All procedures were approved by the Institutional Review Board with a waiver of parental permission for participants ages 16-17 (Mustanski, 2011). At each visit, participants were compensated $\$ 50$.

The current study used data from the first four study visits. Data were available for $850 \mathrm{HIV}-$ negative participants, all of whom reported being assigned male at birth. We excluded 86 participants from the analytic sample, because they did not identify as male at any visit $(N=$ $55)$, they did not identify as gay or bisexual at any visit $(N=28)$, or they did not report any male or female partners at any visit $(N=4)$. Therefore, the analytic sample included 763 participants who provided data on a total of 3,995 partnerships across visits. Because participants enrolled in the study at different times, they varied in how many visits they had completed at the time of data analysis (102 completed one visit, 208 completed two visits, 180 completed three visits, and 273 completed four visits); analyses made use of all available data. Participants provided data on a median of four total partnerships across visits (maximum $=16$ ) and a median of two partnerships per visit. Demographic characteristics are presented in Table 1. 
Demographics-At the baseline visit, participants were asked to report their date of birth, race/ethnicity, and highest level of education. Additionally, they were asked about lifetime HIV/STI testing, pre-exposure prophylaxis (PrEP) use, and self-reported STI diagnoses. Specifically, they were asked: (1) "Have you ever been tested for HIV?" ( $0=$ no, $1=$ yes); (2) "In your entire life, have you ever been tested for STIs such as gonorrhea, chlamydia, syphilis, etc.?" ( $0=$ no, $1=$ yes); (3) "In your entire life, have you ever taken any preexposure prophylaxis (PrEP) medication such as Truvada to reduce your risk of HIV transmission?" ( $0=$ no, $1=$ yes); and (4) "In your entire life, have you ever tested positive for any STI?" ( $0=$ no, $1=$ yes). Other demographics (gender identity and sexual orientation) were assessed at each visit. HIV-status was determined based on self-report and HIV testing. At each visit, participants self-reported their HIV-status and HIV-negative participants received HIV testing. Most participants in the analytic sample self-reported being HIVnegative and received a non-reactive HIV test result at each visit. However, a small number of participants in the analytic sample self-reported being HIV negative but received a reactive HIV test result at a visit ( $N=0-15$ participants or $0.0-2.1 \%$ of participants at each visit). These participants were coded as being HIV-negative at that visit because their perceived HIV-status during the prior six months was most relevant to their sexual behavior during that time frame. At subsequent visits, these participants were coded as being HIVpositive because they knew that they were HIV-positive from that point forward.

HIV risk behaviors-At each visit, participants completed the HIV-Risk Assessment for Sexual Partnerships (H-RASP; Swann, Newcomb, \& Mustanski, 2017). The H-RASP is a self-administered, computerized interview designed to assess sexual behavior and associated situational/contextual variables at the level of the sexual partnership. Sexual risk behavior data collected using the H-RASP has good correspondence with data collected using daily and weekly diaries (Swann et al., 2017) and network interviews (Hogan et al., 2016). In this study, participants reported on up to four sexual partnerships during the preceding six months. For each sexual partnership, participants reported on partner/relationship characteristics, substance use before sex, and condomless sex.

For partner gender, participants were asked: "How does [partner name] currently identify their gender?" Response options included: (1) male; (2) female; (3) male assigned at birth with a different gender identity (such as male-to-female transgender [MTF], transwoman, or genderqueer); and (4) female assigned at birth with a different gender identity (such as female-to-male transgender [FTM], transman, or genderqueer).

For relationship type, participants were asked: "What was your relationship with [partner name]?" Response options included: (1) serious relationship (boyfriend/girlfriend or someone you dated for a while and feel very close to); (2) casually dating but not serious; (3) sleeping with this person but not dating or in a relationship (e.g., fuck buddy or booty call); (4) one night stand (someone you know, but only had sex with once); and (5) stranger or anonymous person (someone you don't know and only had sex with once). Responses were dichotomized for analyzes $(0=$ any non-serious [casual] relationship, $1=$ serious relationship). 
For partner HIV status, participants were asked: "What was the HIV status of [partner name] the last time you had sex?" Response options included: (1) They were HIV negative; (2) They were HIV positive; and (3) I don't know their HIV status. Participant and partner HIV status were combined to create a variable representing HIV concordance $(0=$ discordant, $1=$ concordant). If the participant and the partner had the same HIV status (i.e., they were both HIV-negative or they were both HIV-positive), then they were coded as 1 for HIV concordant. If the participant and the partner had different HIV statuses (i.e., one partner was HIV negative and the other partner was HIV positive), then they were coded as 0 for HIV discordant. If the partner was of unknown HIV status, then they were coded as 0 for HIV discordant.

HIV-negative participants were also asked about their PrEP use with each partner. Specifically, they were asked: "During the past six months when you were having anal or vaginal sex with [partner name], were you taking any pre-exposure prophylaxis (PrEP) medication such as Truvada to reduce your risk of HIV transmission?" $(0=$ no, $1=$ yes $)$.

Condomless sex was assessed separately for insertive and receptive anal sex (for male partners) and for vaginal and anal sex (for female partners). First, participants were asked: "How many times did you have [vaginal/anal] sex with [partner name] during the past six months?" Then, participants were asked: “Of the [number] time(s) you've had [vaginal/anal] sex with [partner name], how many of these times did you have sex without using a condom?" To reduce the impact of outliers, values greater than three standard deviations above the mean were Winsorized (i.e., transformed) to 77 for number of insertive CAS acts with male partners, 70 for number of receptive CAS acts with male partners, 155 for number of condomless vaginal sex acts with female partners, and 34 for number of CAS acts with female partners. This affected less than $2.0 \%$ of the data for each variable.

Substance use before sex was assessed separately for alcohol, marijuana, cocaine, ecstasy, and methamphetamine. For each sexual partnership over the past six months (for up to four sexual partnerships), participants were asked: "How frequently did you use [substance] before having vaginal or anal sex with [partner name] in the past six months?" Response options included: (1) never; (2) less than half the time; (3) about half the time; (4) more than half the time; and (5) always. Responses were dichotomized for analyses $(0=$ never, $1=$ less than half the time, about half the time, more than half the time, or always). Alcohol and marijuana use before sex were included in analyses, but cocaine, ecstasy, and methamphetamine use before sex were not included in analyses because of low endorsement (i.e., cocaine, ecstasy, and methamphetamine were used during $1.8 \%, 1.7 \%$, and $0.6 \%$, respectively, all of sexual partnerships).

\section{Analyses}

All analyses were conducted in R version 3.4 using the 'glmmTMB' package (Magnusson et al., 2016; R Core Team, 2017). Analyses used the partner-level data from all available visits. Partnerships (level 1) were nested within participants (level 2). Mixed effects modeling was used to account for the nesting of partnerships within participants. For condomless sex outcomes, which were count variables, we used negative binomial regression to account for 
the skewed and overdispersed nature of the data. Results are presented as incidence rate ratios (IRRs), which represent the change in the rate of the outcome for each one-unit change in the predictor. For substance use before sex outcomes, which were dichotomous variables, we used logistic regression. Results are presented as odds ratios (ORs), which represent the change in the odds of the outcome for each one-unit change in the predictor. Because data were collected at multiple time points, following the convention of other partner-level analyses (Mustanski, Starks, \& Newcomb, 2014), partnerships that had been reported at prior visits were flagged as repeat partnerships and this variable was included as a covariate at time points after the partner was first reported. Data were included from all partnerships during which each participant reported a male gender identity and a gay or bisexual sexual orientation. Given that participants could report different gender identities and sexual orientations at each visit, these variables were allowed to vary by time point. As such, a given participant's data from a given visit was excluded if they reported a gender identity other than male or a sexual orientation other than gay or bisexual. All analyses comparing gay and bisexual men on sexual behavior (condomless sex and substance use before sex) focused on sex with male partners. Female partnerships were excluded from these analyses because only 10 gay men (1.8\%) compared to 85 bisexual men (40.9\%) reported any female partners at any visits. Transgender partnerships were also excluded from analyses because only 14 gay men (2.5\%) and 27 bisexual men (13.0\%) reported any transgender partners at any visits. Given that our analyses included multiple main effects, interaction effects, and covariates, we did not have sufficient statistical power to examine predictors of sexual behavior with transgender partners for only 14 gay men and 27 bisexual men. Further, there was substantial heterogeneity among the transgender partners (e.g., this group included transgender men, transgender women, and non-binary individuals), which limited the extent to which we could draw meaningful conclusions about sexual behavior with transgender partners.

In the full sample, we tested four models. Models 1 and 2 tested predictors of insertive and receptive CAS acts, including sexual orientation, relationship type, alcohol use before sex, marijuana use before sex, and the interactions between sexual orientation and each of the other predictors (i.e., sexual orientation by relationship type, sexual orientation by alcohol use before sex, and sexual orientation by marijuana use before sex). Models 3 and 4 tested predictors of alcohol and marijuana use before sex, including sexual orientation, relationship type, and their interaction. All of these models focused on male partnerships and adjusted for participant age, race/ethnicity, education, PrEP use, partnership HIV concordance, and whether or not the partnership was a repeat partnership. When an interaction effect was significant, we compared the adjusted means of the outcome variable across the relevant groups. The means were adjusted to account for the covariates in the model. For example, if there was a significant interaction between sexual orientation and relationship type predicting insertive CAS acts, then we would compare the adjusted means of insertive CAS acts across the four combinations of sexual orientation and relationship type (gay and serious partnership, gay and casual partnership, bisexual and serious partnership, and bisexual and casual partnership).

In the subsample of bisexual men, we tested an additional three models. Model 1 tested predictors of condomless sex acts, including partner gender, relationship type, alcohol use 
before sex, marijuana use before sex, and the interactions between partner gender and each of the other predictors (i.e., partner gender by relationship type, partner gender by alcohol use before sex, and partner gender by marijuana use before sex). Models 2 and 3 tested predictors of alcohol and marijuana use before sex, including partner gender, relationship type, and their interaction. All of these models included male and female partnerships, and adjusted for participant age, race/ethnicity, education, PrEP use, partnership HIV concordance, and whether or not the partnership was a repeat partnership.

\section{Results}

Prior to conducting the partner-level analyses, we used baseline data to compare gay and bisexual men on demographic characteristics (age, race/ethnicity, and highest level of education completed) as well as lifetime HIV/STI testing, PrEP use, and self-reported STI diagnoses. Because we used baseline data rather than partner-level data for these analyses, participants were coded as bisexual if they identified as bisexual at any visit. Results are presented in Table 1. Bisexual men were significantly less likely to report ever having an HIV test and ever using PrEP compared to gay men. In contrast, gay and bisexual men did not significantly differ on the odds of ever having an STI test or ever having a self-reported STI diagnosis.

\section{Partner-level analyses}

Next, in the full sample, we examined predictors of insertive and receptive CAS acts and substance use before sex with male partners. Results are presented in Tables 2-4.

Insertive condomless anal sex-The main effect of sexual orientation was not significant. In contrast, there was a significant main effect of relationship type, indicating that the rate of insertive CAS acts was higher for serious partners compared to casual partners. There was also a significant sexual orientation by relationship type interaction (see Figure 1, Panel A). Further examination of this interaction indicated that the rate of insertive CAS acts with casual partners was significantly higher for bisexual men compared to gay men (IRR $=2.07, p=.001$ ), but the rate of insertive CAS acts with serious partners was not significantly different for bisexual men compared to gay men (IRR $=.65, p=.07$ ). Of note, the rate of insertive CAS acts was higher for serious partners compared to casual partners regardless of sexual orientation. Additionally, there were significant main effects of alcohol and marijuana use before sex, indicating that alcohol and marijuana use before sex were both associated with higher rates of insertive CAS acts. Further, there was a significant sexual orientation by marijuana use interaction (see Figure 1, Panel B). The association between marijuana use before sex and insertive CAS acts was stronger for bisexual men (IRR $=6.39, p<.001)$ compared to gay men (IRR $=2.01, p<.001)$, but it was significant for both groups. The sexual orientation by alcohol use interaction was not significant.

Receptive condomless anal sex-The main effect of sexual orientation was not significant. In contrast, there was a significant main effect of relationship type, indicating that the rate of receptive CAS acts was higher with serious partners compared to casual partners. There was also a significant sexual orientation by relationship type interaction (see 
Figure 1, Panel C). Further examination of the interaction indicated that the rate of receptive CAS acts with serious partners was significantly lower for bisexual men compared to gay men $($ IRR $=.49, p=.001)$, but the rate of receptive CAS acts with casual partners was not significantly different for bisexual men compared to gay men (IRR $=.99, p=.97$ ). Of note, the rate of receptive CAS acts was higher with serious partners compared to casual partners regardless of sexual orientation. Additionally, there were significant main effects of alcohol and marijuana use before sex, indicating that alcohol and marijuana use were both associated with higher rates of receptive CAS acts regardless of sexual orientation (i.e., the sexual orientation by alcohol and marijuana use before sex interactions were not significant).

Alcohol and marijuana use before sex-The main effect of sexual orientation on alcohol use before sex was not significant, indicating that the odds of alcohol use before sex did not differ between gay and bisexual men. In contrast, there was a significant main effect of sexual orientation on marijuana use before sex, indicating that the odds of marijuana use before sex were higher for bisexual men compared to gay men. Additionally, there was a significant main effect of relationship type in both models, indicating that the odds of alcohol and marijuana use before sex were higher with serious partners compared to casual partners. The sexual orientation by relationship type interaction was not significant in either model.

\section{Bisexual men}

Finally, in the subsample of bisexual men, we examined predictors of condomless sex and substance use before sex. These analyses included both male and female partnerships. Results are presented in Tables 2, 5, and 6.

Condomless sex-The main effect of partner gender was not significant. There was a significant main effect of relationship type, indicating that the rate of condomless sex acts was higher with serious partners compared to casual partners regardless of partner gender (i.e., the partner gender by relationship type interaction was not significant). Additionally, there were significant main effects of alcohol and marijuana use before sex, indicating that alcohol and marijuana use before sex were associated with higher rates of condomless sex acts regardless of partner gender (i.e., the partner gender by alcohol and marijuana use before sex interactions were not significant).

Alcohol and marijuana use before sex-There was a significant main effect of partner gender on alcohol and marijuana use before sex, indicating that the odds of alcohol and marijuana use before sex were higher with female partners compared to male partners. There was also a significant main effect of relationship type on alcohol and marijuana use before sex, indicating that the odds of alcohol and marijuana use before sex were higher with serious partners compared to casual partners. Further, there was a significant partner gender by relationship type interaction in the alcohol use before sex model (see Figure 1, Panel D). Further examination of this interaction indicated that the odds of alcohol use before sex were higher with serious male partners compared to casual male partners $(\mathrm{OR}=3.02, p<.001)$, but the odds of alcohol use before sex with were not significantly different with serious female partners compared to casual female partners $(\mathrm{OR}=2.28, p=.08)$. Of note, the odds 
of alcohol use before sex were higher with female partners compared to male partners regardless of relationship type. The partner gender by relationship type interaction was not significant in the marijuana use model.

Given that the odds of alcohol and marijuana use before sex were higher with female partners compared to male partners, and that alcohol and marijuana use before sex were associated with condomless sex regardless of partner gender, we conducted a post-hoc analysis to examine the association between partner gender and condomless sex in a model that did not include alcohol and marijuana use before sex. We conducted this analysis to test the possibility that there was an association between partner gender and condomless sex, but that alcohol and marijuana use before sex were accounting for it. The main effect of partner gender on condomless sex was significant in this model, indicating that the rate of condomless sex acts was higher with female partners compared to male partners (IRR = $1.74, p=.008)$. Given that the main effect of partner gender on condomless sex was not significant in the original model, this follow-up finding suggests that alcohol and marijuana use before sex may account for the association between partner gender and condomless sex (i.e., the association between partner gender and condomless sex becomes non-significant controlling for alcohol and marijuana use before sex).

\section{Discussion}

The overarching goal of the current study was to understand risk for HIV among selfidentified bisexual men. Previous research examining differences in HIV risk behaviors between self-identified gay and bisexual men has produced inconsistent findings, in part, due to methodological differences across studies. To overcome limitations of previous research, we used four waves of partner-level data spanning 24-months from a cohort of young MSM. Overall, there were few differences in HIV risk behaviors between selfidentified gay and bisexual young men. However, compared to self-identified gay men, selfidentified bisexual men reported more insertive CAS with casual male partners, they were more likely to report marijuana use before sex with male partners, and they were less likely to report lifetime HIV testing and PrEP use.

\section{Self-identified gay versus bisexual men}

Previous research on differences in condom use between self-identified gay versus bisexual men has led to mixed findings. This is due, in part, to whether or not studies differentiated between serious versus casual partners and insertive versus receptive CAS. We found that differences in CAS between self-identified gay versus bisexual men depended on the type of relationship (serious versus casual) and the type of CAS (insertive versus receptive). Consistent with Agronick et al. (2004), self-identified bisexual men reported more insertive CAS acts with casual male partners compared to self-identified gay men. In contrast, insertive CAS with serious male partners did not differ between self-identified gay and bisexual men. This may be due to the fact that both groups reported more insertive CAS with serious partners compared to casual partners, which is consistent with previous research (for a review, see B. S. Mustanski et al., 2011). This also demonstrates that the higher rate of insertive CAS acts with serious partners compared to casual partners found in previous 
research extends to both self-identified gay and bisexual men. Also consistent with Agronick et al. (2004), self-identified bisexual men reported fewer receptive CAS acts with serious male partners compared to self-identified gay men. In contrast, receptive CAS with casual male partners did not differ between self-identified gay and bisexual men. Of note, the mean number of receptive CAS acts with casual partners was low for both self-identified gay and bisexual men.

Previous research has demonstrated that self-identified bisexual young men were more likely to report substance use before sex with male partners compared to self-identified gay young men (Agronick et al., 2004). We found that self-identified bisexual men were more likely to report marijuana use before sex with male partners, but not alcohol use, compared to selfidentified gay men. Our data cannot explain why this finding was specific to marijuana use, but it may be due to different expectations for marijuana versus alcohol. There is some evidence that people use marijuana to enhance their perceptual experiences more often than alcohol, whereas they use alcohol to facilitate social interactions more often than marijuana (Simons, Correia, \& Carey, 2000). In a sample of young MSM, bisexual men reported using marijuana to enhance pleasure more often than gay men, but this did not extend to alcohol (Feinstein \& Newcomb, 2016). Therefore, although speculative, self-identified bisexual men may be particularly likely to use marijuana before sex due to expectations that it will enhance their experiences, but this may not extend to alcohol due to different expectations. It will be important for future research to examine the extent to which substance use motivations and norms explain differences in substance use before sex between selfidentified gay and bisexual men.

Regardless, both alcohol and marijuana use before sex were associated with greater insertive and receptive CAS with male partners for both self-identified gay and bisexual men, and the association between marijuana use and insertive CAS was stronger for self-identified bisexual men compared to self-identified gay men. Given that both risk behaviors (marijuana use before sex and insertive CAS with casual male partners) were higher among selfidentified bisexual men compared to self-identified gay men, it is possible that self-identified bisexual men's use of marijuana reduces their inhibitions and decision-making capabilities, leading to reduced condom use. Internalized stigma may also prevent self-identified bisexual men from effectively negotiating condom use with partners, especially when using marijuana. It will be important for future research to examine the extent to which stigmarelated experiences and marijuana use motivations and expectations influence CAS among self-identified bisexual men.

\section{Self-identified bisexual men}

One of the novel aspects of the current study was our ability to examine differences in HIV risk behaviors with male versus female partners among self-identified bisexual men. A previous study found that self-identified bisexual adult men were less likely to report consistent condom use with female partners compared to male partners (Stokes et al., 1993), but that study did not examine within-person associations. In contrast, we did not find a significant difference between condomless sex with female versus male partners in our sample of self-identified bisexual young men. However, our model examining the 
association between partner gender and condomless sex controlled for the effects of alcohol and marijuana use before sex. Given that the odds of alcohol and marijuana use before sex were higher with female partners compared to male partners, and that both were associated with condomless sex regardless of partner gender (discussed below), we conducted a posthoc analysis to examine the association between partner gender and condomless sex without controlling for these effects. The post-hoc analysis demonstrated that self-identified bisexual men reported more condomless sex acts with female partners compared to male partners, suggesting that substance use before sex may account for the association between partner gender and condomless sex that was found in a previous study (Stokes et al., 1993). These findings highlight the importance of accounting for confounding risk factors when examining differences in sexual risk behavior with male versus female partners.

Previous qualitative research has found that behaviorally bisexual adult men, many of whom also self-identify as bisexual, report unique barriers to using condoms with both male and female partners (Dodge et al., 2008; Hubach et al., 2014; Munoz-Laboy \& Dodge, 2007). Therefore, it is possible that self-identified bisexual young men's condom use does not differ with male versus female partners (after accounting for substance use before sex), but their reasons for not using condoms with male versus female partners may differ. Of note, Mustanski, Ryan, and Garofalo (2014) found that condom use errors were common among young MSM, and some errors were more common during vaginal sex compared to anal sex. However, in the subsample of behaviorally bisexual young men, condom use errors did not differ between anal and vaginal sex. Based on these findings, young bisexual men may benefit from interventions that build motivation and skills for correct condom use with both male and female partners.

We also found that self-identified bisexual men were more likely to report alcohol and marijuana use before sex with female partners compared to male partners. This is in contrast to previous findings that behaviorally bisexual adult men were more likely to use alcohol with male sexual partners compared to female sexual partners (Martinez et al., 2012), and that behaviorally bisexual men as well as non-gay-identifying MSM described using alcohol with male sexual partners to feel more comfortable having sex with other men (Harawa et al., 2008; Martinez et al., 2012). Given that all of the bisexual men in our sample selfidentified as bisexual and were young (ages 16-29), they may have been more comfortable having sex with other men compared to the older behaviorally bisexual men and non-gayidentifying MSM in the previous studies. Although our data cannot explain why the selfidentified bisexual men in our sample were more likely to report alcohol and marijuana use before sex with female partners compared to male partners, we offer two potential explanations.

As noted, there is evidence that behaviorally bisexual adult men perceive female partners as posing less risk for HIV compared to male partners (Dodge et al., 2008; Hubach et al., 2014). Although this has been described as a barrier to bisexual men using condoms with female partners, it may also contribute to bisexual men being less concerned about being disinhibited during sex with female partners. However, there is also evidence that behaviorally bisexual adult men perceive female partners as being less accepting of their bisexual orientation compared to male partners (Dodge et al., 2008). As such, it is also 
possible that bisexual men are less comfortable during sex with female partners (e.g., due to concerns about acceptance and/or internalized stigma), in which case they may be more likely to rely on the disinhibiting effects of substance use before sex with female partners. Regardless of partner gender, alcohol and marijuana use before sex were both associated with more condomless sex. Therefore, alcohol and marijuana use before sex represent risk factors for condomless sex with both male and female partners among self-identified bisexual young men.

\section{Implications for HIV prevention}

The current findings have important implications for HIV prevention. Scholars have suggested that bisexual men may be less likely to receive HIV prevention information than gay men due to low levels of integration and acceptance in the gay community (Doll, Myers, Kennedy, \& Allman, 1997; Kennedy \& Doll, 2001). Although few studies have compared self-identified gay and bisexual men on HIV/STI testing and PrEP use, there is some evidence that self-identified bisexual men are less likely to get tested for HIV/STIs (Jeffries, 2010; Jin et al., 2002) and to use PrEP (Grov, Rendina, Jimenez, \& Parsons, 2016) compared to self-identified gay men. Consistent with these findings, we also found that self-identified bisexual young men had lower odds of ever having an HIV test and lower odds of ever using PrEP compared to self-identified gay young men. These findings suggest that self-identified bisexual young men are less likely to access HIV prevention services compared to selfidentified gay young men, highlighting the need to increase HIV prevention focused on selfidentified bisexual men.

At present, there are no evidence-based HIV prevention interventions specifically for selfidentified bisexual men. In fact, in 2015, the CDC developed a list of 84 evidence-based behavioral interventions for HIV prevention, but only $11 \%$ were for MSM, $2 \%$ for young MSM, and none specifically for bisexual men (Hergenrather et al., 2016). Meanwhile, it has been suggested that existing HIV prevention interventions may be less relevant for bisexual men compared to gay men because of their focus on receptive anal sex with male partners and their lack of attention to vaginal sex with female partners and oral sex with partners of different genders (Dodge, Van Der Pol, et al., 2012). Further, self-identified bisexual individuals report negative experiences with healthcare providers (e.g., dismissing bisexuality as a phase, assuming that they are promiscuous) (Eady, Dobinson, \& Ross, 2011; Ontario Public Health Association, 2003; Page, 2004), highlighting the potential value of an intervention specifically for self-identified bisexual men.

There have been several attempts to develop HIV prevention interventions for behaviorally bisexual men (Jeffries, 2014), which generally focus on reducing HIV risk behaviors and promoting protective behaviors, while attending to the unique sociocultural influences on these behaviors among behaviorally bisexual men. However, these interventions do not address the unique concerns of self-identified bisexual men. Having a bisexual identity may expose an individual to additional stressors (e.g., rejection or discrimination from both gay and heterosexual individuals, internalizing negative societal attitudes toward bisexuality), and these experiences may confer risk for engaging in condomless sex and other HIV-related risk behaviors. For these reasons, researchers have emphasized the need for interventions to 
provide comprehensive sexual health education, while teaching skills to cope with stigma and to promote acceptance of one's bisexual identity (Dodge, Schnarrs, et al., 2012; Dodge et al., 2013; Hubach et al., 2014; Malebranche et al., 2010; Ross, Dobinson, \& Eady, 2010). Our findings highlight the need for HIV prevention interventions for self-identified bisexual men to focus on increasing HIV/STI testing and PrEP use, reducing insertive CAS and marijuana use before sex with male partners, and addressing their unique risk for condomless sex and substance use before sex with female partners. Our findings also highlight the critical need to address substance use before sex as a risk factor for condomless sex with male and female partners.

These findings should be interpreted in the context of several important limitations. First, because participants could report different gender identities and sexual orientations across visits, we treated these as time-varying in analyses. While findings can still be interpreted as differences between self-identified gay and bisexual men, it is possible that changes in gender identity and sexual orientation have an influence on HIV risk behaviors. In fact, there is some evidence that changes in self-identified sexual orientation are associated with increased substance use, although these associations are stronger for females (compared to males) and adolescents (compared to young adults) (Ott et al., 2013). It will be important for future research to test the extent to which changes in self-identified sexual orientation are associated with HIV risk behaviors. Second, we did not have sufficient statistical power to examine predictors of sexual behavior with transgender partners because only 14 gay men and 27 bisexual men reported any transgender partners at any visits. Previous research has demonstrated that transgender women are also at increased risk for HIV (Baral et al., 2013; Herbst et al., 2008). As such, it will be important for future research to continue to examine differences in HIV risk behaviors with partners of different genders, including transgender individuals, to fully understand risk for HIV among self-identified gay and bisexual men.

Third, participants reported on their sexual behavior at the partner-level, but not at the eventlevel. Partner-level analyses represent a substantial strength over designs that focus on a single partnership or aggregate across partnerships, but it remains unclear if findings extend to specific sexual encounters (i.e., the event-level). Fourth, although we accounted for participant PrEP use and HIV concordance between partners, we did not account for participant adherence to PrEP or partner PrEP use. Given that adherence to PrEP is critical to its efficacy, and that both partners' use of biomedical prevention can influence condom use, it will be important for future research to examine how these factors influence condom use decisions among self-identified gay and bisexual men. Finally, despite a large, racially/ ethnically diverse sample, all participants were from the Chicago area and findings may not generalize to young MSM in other locations.

Despite these limitations, the current findings are among the first to compare self-identified gay and bisexual young men on HIV risk behaviors and to examine partner gender as a predictor of HIV risk behaviors among self-identified bisexual young men. Findings revealed a unique risk profile among self-identified bisexual men, including more insertive CAS with casual male partners, greater odds of marijuana use before sex with male partners, and lower odds of HIV testing and PrEP use compared to gay young men, as well as greater odds of alcohol and marijuana use before sex with female partners compared to male 
partners. There is a critical need for research focused on why self-identified gay and bisexual men differ on certain HIV risk behaviors, and why self-identified bisexual men are more likely to use alcohol and marijuana before sex with female partners. Throughout our discussion, we offer several potential explanations for these findings, many of which involve stigma-related experiences (e.g., concerns about acceptance, internalized stigma). Previous research has demonstrated that bisexual individuals experience unique stigma-related stressors compared to other sexual minorities, such as rejection and discrimination from both heterosexual and gay/lesbian individuals (Feinstein \& Dyar, 2017). Further, bisexual individuals are less likely to be open about their sexual orientation compared to gay/lesbian individuals, and being open about one's sexual orientation can put bisexual individuals at risk for discrimination and negative health outcomes (Feinstein, Dyar, \& London, 2016). As such, it will be important for future research to examine the extent to which stigma-related experiences influence sexual behavior among bisexual individuals. Continued research on HIV risk behaviors among self-identified bisexual men is critical to informing the development of HIV prevention interventions for this population.

\section{References}

Agronick G, O’Donnell L, Stueve A, Doval AS, Duran R, Vargo S. 2004; Sexual behaviors and risks among bisexually- and gay-identified young Latino men. AIDS Behav. 8(2):185-197. DOI: 10.1023/B:AIBE.0000030249.11679.d0 [PubMed: 15187480]

Arnett JJ. 2000; Emerging adulthood. A theory of development from the late teens through the twenties. American Psychologist. 55(5):469-480. DOI: 10.1037/0003-066X.55.5.469 [PubMed: 10842426]

Balsam KF, Mohr JJ. 2007; Adaptation to sexual orientation stigma: A comparison of bisexual and lesbian/gay adults. Journal of Counseling Psychology. 54:306-319.

Baral SD, Poteat T, Stromdahl S, Wirtz AL, Guadamuz TE, Beyrer C. 2013; Worldwide burden of HIV in transgender women: a systematic review and meta-analysis. Lancet Infect Dis. 13(3):214-222. DOI: 10.1016/S1473-3099(12)70315-8 [PubMed: 23260128]

Bauer GR, Brennan DJ. 2013; The problem with "behavioral bisexuality": assessing sexual orientation in survey research. Journal of Bisexuality. 13:148-165.

Carliner H, Mauro PM, Brown QL, Shmulewitz D, Rahim-Juwel R, Sarvet AL, ... Hasin DS. 2017; The widening gender gap in marijuana use prevalence in the U.S. during a period of economic change, 2002-2014. Drug Alcohol Depend. 170:51-58. DOI: 10.1016/j.drugalcdep.2016.10.042 [PubMed: 27875801]

CDC. HIV among gay and bisexual men. 2015. Retrieved from http://www.cdc.gov/hiv/group/msm/ index.html

CDC. HIV Surveillance Report, 2015. 2016. Retrieved from http://www.cdc.gov/hiv/library/reports/ hiv-surveillance.html

Cooper ML. 2010; Toward a person x situation model of sexual risk-taking behaviors: illuminating the conditional effects of traits across sexual situations and relationship contexts. J Pers Soc Psychol. 98(2):319-341. DOI: 10.1037/a0017785 [PubMed: 20085403]

Cox N, Vanden Berghe W, Dewaele A, Vincke J. 2010; Acculturation strategies and mental health in gay, lesbian, and bisexual youth. J Youth Adolesc. 39(10):1199-1210. DOI: 10.1007/ s10964-009-9435-7 [PubMed: 20689983]

Delker E, Brown Q, Hasin DS. 2016; Alcohol Consumption in Demographic Subpopulations: An Epidemiologic Overview. Alcohol Res. 38(1):7-15. [PubMed: 27159807]

Dodge B, Jeffries WLt, Sandfort TG. 2008; Beyond the Down Low: sexual risk, protection, and disclosure among at-risk Black men who have sex with both men and women (MSMW). Arch Sex Behav. 37(5):683-696. DOI: 10.1007/s10508-008-9356-7 [PubMed: 18512140] 
Dodge B, Schnarrs PW, Goncalves G, Malebranche D, Martinez O, Reece M, ... Fortenberry JD. 2012; The significance of privacy and trust in providing health-related services to behaviorally bisexual men in the United States. AIDS Educ Prev. 24(3):242-256. DOI: 10.1521/aeap. 2012.24.3.242 [PubMed: 22676463]

Dodge B, Schnarrs PW, Reece M, Martinez O, Goncalves G, Malebranche D, ... Fortenberry JD. 2013; Sexual behaviors and experiences among behaviorally bisexual men in the midwestern United States. Arch Sex Behav. 42(2):247-256. DOI: 10.1007/s10508-011-9878-2 [PubMed: 22187027]

Dodge B, Van Der Pol B, Reece M, Malebranche D, Martinez O, Goncalves G, ... Fortenberry JD. 2012; Rectal self-sampling in non-clinical venues for detection of sexually transmissible infections among behaviourally bisexual men. Sex Health. 9(2):190-191. DOI: 10.1071/SH11068 [PubMed: 22498165]

Doll L, Myers T, Kennedy M, Allman D. 1997; Bisexuality and HIV risk: experiences in Canada and the United States. Annu Rev Sex Res. 8:102-147. [PubMed: 10051892]

Doll LS, Beeker C. 1996; Male bisexual behavior and HIV risk in the United States: synthesis of research with implications for behavioral interventions. AIDS Educ Prev. 8(3):205-225. [PubMed: 8806950]

Doll LS, Petersen LR, White CR, Johnson ES, Ward JW. The Blood Donor Study Group. 1992; Homosexually and nonhomosexually identified men who have sex with men: A behavioral comparison. The Journal of Sex Research. 29:1-14.

Drumright LN, Patterson TL, Strathdee SA. 2006; Club drugs as causal risk factors for HIV acquisition among men who have sex with men: a review. Subst Use Misuse. 41(10-12):15511601. DOI: 10.1080/10826080600847894 [PubMed: 17002993]

Duncan SC, Duncan TE, Hops H. 1996; Analysis of longitudinal data within accelerated longitudinal designs. Psychological Methods. 1:236-248.

Dunlap A. 2016; Changes in coming out milestones across five age cohorts. Journal of Gay \& Lesbian Social Services. 28:20-38.

Eady A, Dobinson C, Ross LE. 2011; Bisexual people's experiences with mental health services: a qualitative investigation. Community Ment Health J. 47(4):378-389. DOI: $10.1007 /$ s10597-010-9329-x [PubMed: 20602170]

Everett BG, Schnarrs PW, Rosario M, Garofalo R, Mustanski B. 2014; Sexual orientation disparities in sexually transmitted infection risk behaviors and risk determinants among sexually active adolescent males: results from a school-based sample. Am J Public Health. 104(6):1107-1112. DOI: 10.2105/AJPH.2013.301759 [PubMed: 24825214]

Feinstein BA, Dyar C. 2017; Bisexuality, minority stress, and health. Current Sexual Health Reports. doi: 10.1007/s11930-017-0096-3

Feinstein BA, Dyar C, London B. 2016; Are Outness and Community Involvement Risk or Protective Factors for Alcohol and Drug Abuse Among Sexual Minority Women? Arch Sex Behav. doi: 10.1007/s10508-016-0790-7

Feinstein BA, Newcomb ME. 2016; The role of substance use motives in the associations between minority stressors and substance use problems among young men who have sex with men. Psychol Sex Orientat Gend Divers. 3(3):357-366. DOI: 10.1037/sgd0000185 [PubMed: 27713906]

Friedman MR, Wei C, Klem ML, Silvestre AJ, Markovic N, Stall R. 2014; HIV infection and sexual risk among men who have sex with men and women (MSMW): a systematic review and metaanalysis. PLoS One. 9(1):e87139.doi: 10.1371/journal.pone.0087139 [PubMed: 24498030]

Grant BF, Dawson DA, Stinson FS, Chou SP, Dufour MC, Pickering RP. 2004; The 12-month prevalence and trends in DSM-IV alcohol abuse and dependence: United States, 1991-1992 and 2001-2002. Drug Alcohol Depend. 74(3):223-234. DOI: 10.1016/j.drugalcdep.2004.02.004 [PubMed: 15194200]

Grov C, Rendina HJ, Jimenez R, Parsons JT. 2016; Using Online Settings to Identify Gay and Bisexual Men Willing to Take or With Experience Taking PrEP: Implications for Researchers and Providers. AIDS Educ Prev. 28(5):378-392. DOI: 10.1521/aeap.2016.28.5.378 [PubMed: 27710085] 
Harawa NT, Williams JK, Ramamurthi HC, Manago C, Avina S, Jones M. 2008; Sexual behavior, sexual identity, and substance abuse among low-income bisexual and non-gay-identifying African American men who have sex with men. Arch Sex Behav. 37(5):748-762. DOI: 10.1007/ s10508-008-9361-x [PubMed: 18546069]

Herbst JH, Jacobs ED, Finlayson TJ, McKleroy VS, Neumann MS, Crepaz N. HIV/AIDS Prevention Research Synthesis Team. 2008; Estimating HIV prevalence and risk behaviors of transgender persons in the United States: a systematic review. AIDS and Behavior. 12(1):1-17. DOI: 10.1007/ s10461-007-9299-3 [PubMed: 17694429]

Hergenrather KC, Emmanuel D, Durant S, Rhodes SD. 2016; Enhancing HIV Prevention Among Young Men Who Have Sex With Men: A Systematic Review of HIV Behavioral Interventions for Young Gay and Bisexual Men. AIDS Educ Prev. 28(3):252-271. DOI: 10.1521/aeap. 2016.28.3.252 [PubMed: 27244193]

Herrick AL, Marshal MP, Smith HA, Sucato G, Stall RD. 2011; Sex while intoxicated: a meta-analysis comparing heterosexual and sexual minority youth. J Adolesc Health. 48(3):306-309. DOI: 10.1016/j.jadohealth.2010.07.008 [PubMed: 21338904]

Hogan B, Melville JR, Philips GL 2nd, Janulis P, Contractor N, Mustanski BS, Birkett M. 2016; Evaluating the Paper-to-Screen Translation of Participant-Aided Sociograms with High-Risk Participants. Proc SIGCHI Conf Hum Factor Comput Syst. 2016:5360-5371. DOI: 10.1145/2858036.2858368 [PubMed: 28018995]

Hubach RD, Dodge B, Goncalves G, Malebranche D, Reece M, Van Der Pol B, ... Fortenberry JD. 2014; Gender matters: condom use and nonuse among behaviorally bisexual men. Arch Sex Behav. 43(4):707-717. DOI: 10.1007/s10508-013-0147-4 [PubMed: 23912775]

Janulis P, Feinstein BA, Phillips G 2nd, Newcomb ME, Birkett M, Mustanski B. 2017; Sexual Partner Typologies and the Association Between Drug Use and Sexual Risk Behavior Among Young Men Who Have Sex With Men. Arch Sex Behav. doi: 10.1007/s10508-016-0909-x

Jeffries, WLt. 2010; HIV testing among bisexual men in the United States. AIDS Educ Prev. 22(4): 356-370. DOI: 10.1521/aeap.2010.22.4.356 [PubMed: 20707695]

Jeffries, WLt. 2014; Beyond the bisexual bridge: sexual health among U.S. men who have sex with men and women. Am J Prev Med. 47(3):320-329. DOI: 10.1016/j.amepre.2014.05.002 [PubMed: 24970239]

Jeffries, WLt; Dodge, B. 2007; Male bisexuality and condom use at last sexual encounter: results from a national survey. J Sex Res. 44(3):278-289. [PubMed: 17879171]

Jessor R. 1992; Risk behavior in adolescence: A psychosocial framework for understanding and action. Developmental Review. 12(4):374-390.

Jin FY, Prestage G, Law MG, Kippax S, Van de Ven P, Rawsthorne P, ... Grulich AE. 2002; Predictors of recent HIV testing in homosexual men in Australia. HIV Med. 3(4):271-276. [PubMed: 12444945]

Kennedy M, Doll LS. 2001; Male bisexuality and HIV risk. Journal of Bisexuality. 1:111-135.

Kessler RC, Berglund P, Demler O, Jin R, Merikangas KR, Walters EE. 2005; Lifetime prevalence and age-of-onset distributions of DSM-IV disorders in the National Comorbidity Survey Replication. Arch Gen Psychiatry. 62(6):593-602. DOI: 10.1001/archpsyc.62.6.593 [PubMed: 15939837]

Lachowsky NJ, Saxton PJ, Hughes AJ, Dickson NP, Summerlee AJ, Milhausen RR, Dewey CE. 2015; Younger Gay and Bisexual Men's Condom Use With Main Regular Sexual Partner in New Zealand. AIDS Educ Prev. 27(3):257-274. DOI: 10.1521/aeap.2015.27.3.257 [PubMed: 26010316]

Lever J, Kanouse DE, Rogers WH, Carson S, Hertz R. 1992; Behavior patterns and sexual identity of bisexual males. The Journal of Sex Research. 29:141-167.

Magnusson, A; Skaug, H; Nielsen, A; Berg, C; Kristensen, K; Maechler, M; ... Brooks, M. glmmTMB: Generalized Linear Mixed Models using Template Model Builder. 2016. Retrieved from https://github.com/glmmTMB/

Malebranche DJ, Arriola KJ, Jenkins TR, Dauria E, Patel SN. 2010; Exploring the "bisexual bridge": a qualitative study of risk behavior and disclosure of same-sex behavior among black bisexual men. Am J Public Health. 100(1):159-164. [PubMed: 19910348] 
Martinez O, Dodge B, Goncalves G, Schnarrs P, Munoz-Laboy M, Reece M, .. Fortenberry JD. 2012; Sexual Behaviors and Experiences among Behaviorally Bisexual Latino Men in the Midwestern United States: Implications for Sexual Health Interventions. J Bisex. 12(2):283-310. DOI: 10.1080/15299716.2012.674865 [PubMed: 22685383]

Martos A, Nezhad S, Meyer IH. 2015; Variations in Sexual Identity Milestones among Lesbians, Gay Men and Bisexuals. Sex Res Social Policy. 12(1):24-33. DOI: 10.1007/s13178-014-0167-4 [PubMed: 27695579]

McKechnie ML, Bavinton BR, Zablotska IB. 2013; Understanding of norms regarding sexual practices among gay men: literature review. AIDS Behav. 17(4):1245-1254. DOI: 10.1007/ s10461-012-0309-8 [PubMed: 22983537]

Munoz-Laboy M, Dodge B. 2007; Bisexual Latino men and HIV and sexually transmitted infections risk: an exploratory analysis. Am J Public Health. 97(6):1102-1106. [PubMed: 17463376]

Mustanski B. 2011; Ethical and regulatory issues with conducting sexuality research with LGBT adolescents: a call to action for a scientifically informed approach. Archives of Sexual Behavior. 40:673-686. DOI: 10.1007/s10508-011-9745-1 [PubMed: 21528402]

Mustanski, B, Kuper, L, Greene, GJ. Development of sexual orientation and identity. In: Tolman, DL, Diamond, LM, editorsHandbook of Sexuality and Psychology. Washington, DC: American Psychological Association; 2014. 597-628.

Mustanski B, Newcomb ME, Clerkin EM. 2011; Relationship characteristics and sexual risk-taking in young men who have sex with men. Health Psychol. 30(5):597-605. DOI: 10.1037/a0023858 [PubMed: 21604883]

Mustanski B, Ryan DT, Garofalo R. 2014; Associations of sexually transmitted infections with condom problems among young men who have sex with men. Sex Transm Dis. 41(7):427-432. DOI: 10.1097/OLQ.0000000000000150 [PubMed: 24922101]

Mustanski B, Starks T, Newcomb ME. 2014; Methods for the design and analysis of relationship and partner effects on sexual health. Archives of Sexual Behavior. 43(1):21-33. DOI: 10.1007/ s10508-013-0215-9 [PubMed: 24243003]

Mustanski B, Swann G, Newcomb ME, Prachand N. 2017Effects of parental monitoring and knowledge on substance use and HIV risk behaviors among young men who have sex with men: Results from three studies. AIDS and Behavior.

Mustanski BS, Newcomb M, Du Bois SN, Garcia SC, Grov C. 2011; HIV in young men who have sex with men: A review of epidemiology, risk and protective factors, and interventions. J Sex Res. 48(2-3):218-253. DOI: 10.1080/00224499.2011.558645 [PubMed: 21409715]

Newcomb ME, Mustanski B. 2013; Racial differences in same-race partnering and the effects of sexual partnership characteristics on HIV Risk in MSM: a prospective sexual diary study. J Acquir Immune Defic Syndr. 62(3):329-333. DOI: 10.1097/QAI.0b013e31827e5f8c [PubMed: 23187943]

Newcomb ME, Mustanski B. 2014; Developmental change in the relationship between alcohol and drug use before sex and sexual risk behavior in young men who have sex with men. AIDS Behav. 18(10):1981-1990. DOI: 10.1007/s10461-014-0766-3 [PubMed: 24696227]

Newcomb ME, Ryan DT, Greene GJ, Garofalo R, Mustanski B. 2014; Prevalence and patterns of smoking, alcohol use, and illicit drug use in young men who have sex with men. Drug Alcohol Depend. 141:65-71. DOI: 10.1016/j.drugalcdep.2014.05.005 [PubMed: 24907774]

Ontario Public Health Association. Improving the access and quality of public health services for bisexuals. 2003. Retrieved from Toronto, Ontario

Ott MQ, Wypij D, Corliss HL, Rosario M, Reisner SL, Gordon AR, Austin SB. 2013; Repeated changes in reported sexual orientation identity linked to substance use behaviors in youth. $\mathrm{J}$ Adolesc Health. 52(4):465-472. DOI: 10.1016/j.jadohealth.2012.08.004 [PubMed: 23298999]

Page EH. 2004; Mental health services experiences of bisexual women and bisexual men: An empirical study. Journal of Bisexuality. 4:137-160.

Parsons JT, Siegel AW, Cousins JH. 1997; Late adolescent risk-taking: effects of perceived benefits and perceived risks on behavioral intentions and behavioral change. J Adolesc. 20(4):381-392. DOI: 10.1006/jado.1997.0094 [PubMed: 9268413] 
R Core Team. R: A language and environment for statistical computing. 2017. Retrieved from https:// www.R-project.org/

Ross LE, Dobinson C, Eady A. 2010; Perceived determinants of mental health for bisexual people: a qualitative examination. Am J Public Health. 100(3):496-502. DOI: 10.2105/AJPH.2008.156307 [PubMed: 20075326]

Siegel, AW, Cuccaro, P, Parsons, JT, Wall, J, Weinberg, AD. Adolescents' thinking about emotions and risk-taking. In: Puckett, JM, Reese, HW, editorsMechanisms of everyday cognition. Hillsdale, N.J: L. Erlbaum Associates; 1993. 155-175.

Simons J, Correia CJ, Carey KB. 2000; A comparison of motives for marijuana and alcohol use among experienced users. Addict Behav. 25(1):153-160. [PubMed: 10708331]

Stokes JP, McKirnan D, Burzette R. 1993; Sexual behavior, condom use, disclosure of sexuality, and stability of sexual orientation in bisexual men. Journal of Sex Research. 30:203-213.

Stokes JP, Vanable P, McKirnan DJ. 1997; Comparing gay and bisexual men on sexual behavior, condom use, and psychosocial variables related to HIV/AIDS. Arch Sex Behav. 26(4):383-397. [PubMed: 9251836]

Swann G, Newcomb ME, Mustanski B. 2017Validation of the HIV Risk Assessment of Sexual Partners (H-RASP): Comparison to a two month prospective diary study. Archives of Sexual Behavior.

Thompson RG Jr, Eaton NR, Hu MC, Grant BF, Hasin DS. 2014; Regularly drinking alcohol before sex in the United States: effects of relationship status and alcohol use disorders. Drug Alcohol Depend. 141:167-170. DOI: 10.1016/j.drugalcdep.2014.05.021 [PubMed: 24950638]

Vosburgh HW, Mansergh G, Sullivan PS, Purcell DW. 2012; A review of the literature on event-level substance use and sexual risk behavior among men who have sex with men. AIDS Behav. 16(6): 1394-1410. DOI: 10.1007/s10461-011-0131-8 [PubMed: 22323004]

Young RM, Meyer IH. 2005; The Trouble With "MSM" and "WSW": Erasure of the Sexual-Minority Person in Public Health Discourse. Am J Public Health. 95(7):1144-1149. DOI: 10.2105/ajph. 2004.046714 [PubMed: 15961753] 

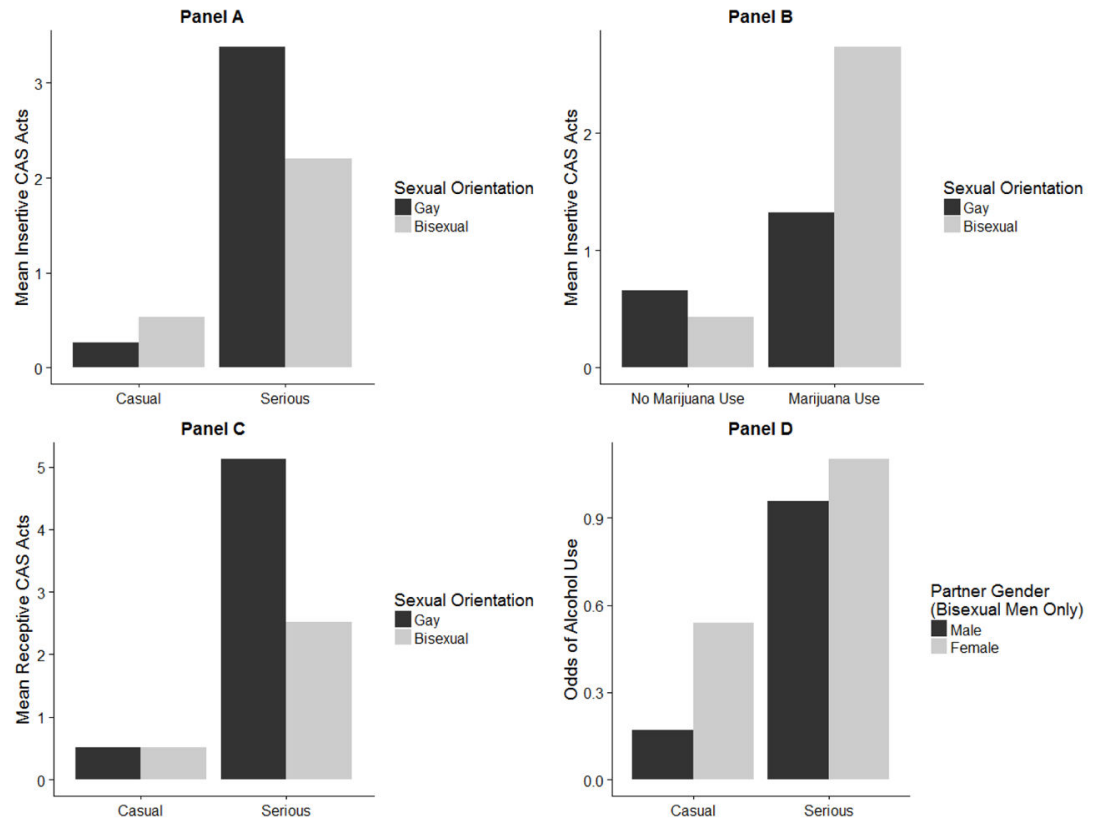

Figure 1.

Examination of significant interactions in the partnership-level analyses. Panel A depicts the association between relationship type and insertive CAS acts as a function of sexual orientation. Panel B depicts the association between marijuana use before sex and insertive CAS acts as a function of sexual orientation. Panel $\mathrm{C}$ depicts the association between relationship type and receptive CAS acts as a function of sexual orientation. Panel D depicts the association between relationship type and alcohol use before sex as a function of partner gender in the subsample of bisexual men. 


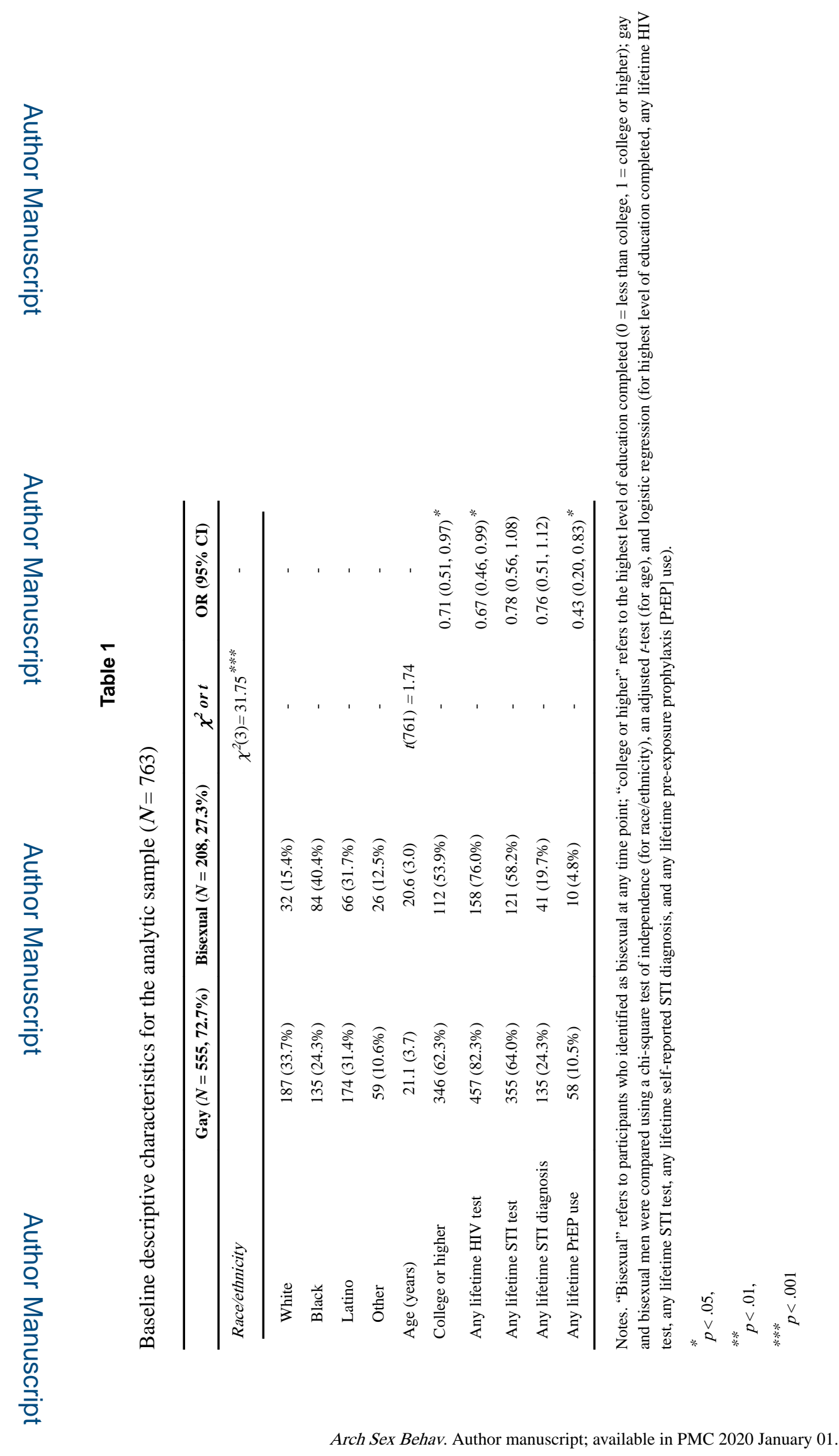




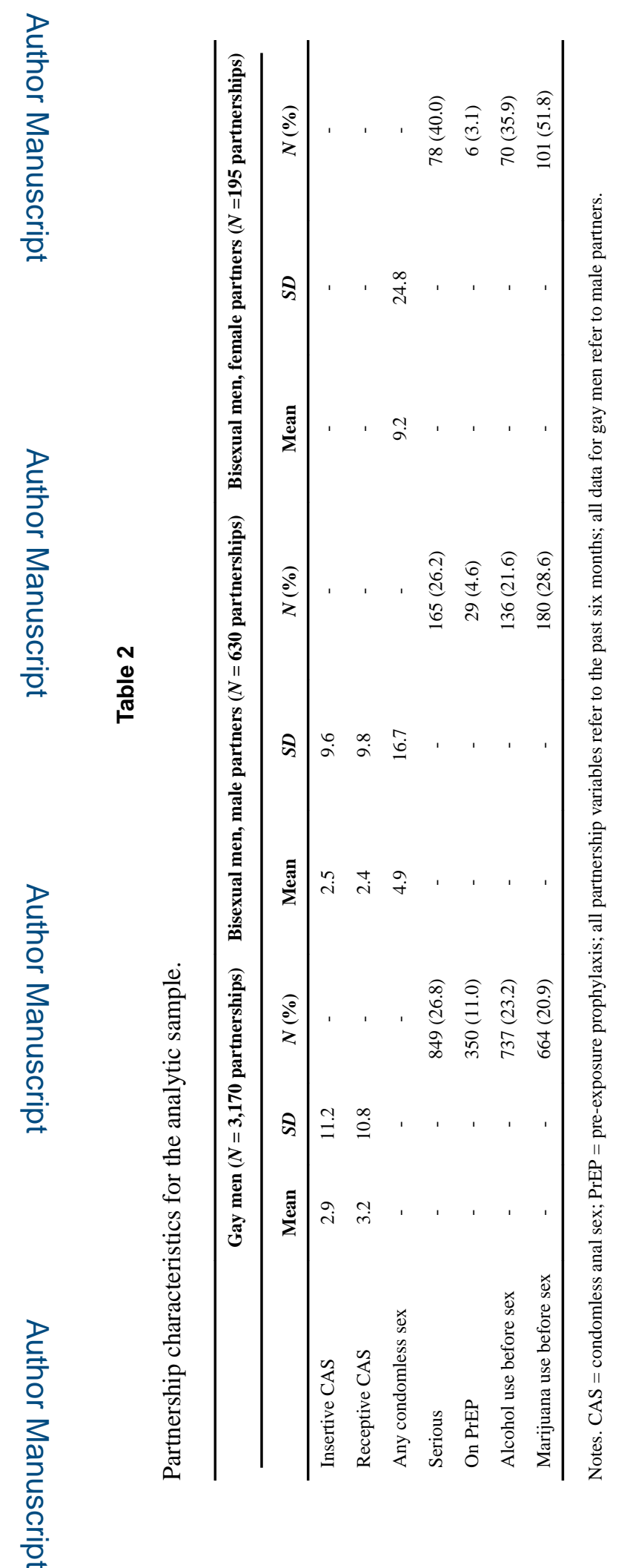

Arch Sex Behav. Author manuscript; available in PMC 2020 January 01. 


\section{Table 3}

Predictors of condomless anal sex acts with male partners in the full sample.

\begin{tabular}{lcc}
\hline Predictor & $\begin{array}{c}\text { Insertive CAS acts } \\
\text { IRR }(95 \% \text { CI })\end{array}$ & $\begin{array}{c}\text { Receptive CAS acts } \\
\text { IRR }(95 \% \text { CI })\end{array}$ \\
\hline Serious & $13.15(10.33,16.75)^{* * * *}$ & $9.95(7.98,12.4)^{* * *}$ \\
Bisexual & $1.29(0.83,2.01)$ & $0.73(0.48,1.11)$ \\
Alcohol & $2.10(1.63,2.70)^{* * *}$ & $2.38(1.9,2.98)^{* * *}$ \\
Marijuana & $2.00(1.55,2.59)^{* * *}$ & $2.11(1.67,2.68)^{* * *}$ \\
Serious x Bisexual & $0.31(0.18,0.55)^{* * *}$ & $0.50(0.29,0.84)^{* *}$ \\
Alcohol x Bisexual & $0.81(0.44,1.49)$ & $1.21(0.70,2.11)$ \\
Marijuana x Bisexual & $3.19(1.76,5.76)^{* * *}$ & $1.52(0.87,2.68)$ \\
\hline
\end{tabular}

Notes. CAS = condomless anal sex; IRR = incidence rate ratio; $\mathrm{CI}=$ confidence interval; all partnership variables refer to the past six months; "serious" refers to the main effect of relationship type $(0=$ casual, $1=$ serious $)$; "bisexual" refers to the main effect of sexual orientation $(0=$ gay, 1 = bisexual); all estimates are adjusted for age, race/ethnicity, education, PrEP use, partnership HIV concordance, and whether or not the partnership was a repeat partnership.

$p<.05$,

*** $p<.01$,

$* * * x<.001$ 


\section{Table 4}

Predictors of substance use before sex with male partners in the full sample.

\begin{tabular}{lcc}
\hline Predictor & $\begin{array}{c}\text { Alcohol use before sex } \\
\text { OR }(95 \% \text { CI })\end{array}$ & $\begin{array}{c}\text { Marijuana use before sex } \\
\text { OR }(95 \% \text { CI })\end{array}$ \\
\hline Serious & $5.86(4.48,7.67)^{* * *}$ & $3.10(2.34,4.12)^{* * *}$ \\
Bisexual & $1.16(0.76,1.79)$ & $2.00(1.26,3.16)^{* *}$ \\
Serious x Bisexual & $1.03(0.58,1.86)$ & $0.96(0.51,1.80)$ \\
\hline
\end{tabular}

Notes. OR = odds ratio; $\mathrm{CI}=$ confidence interval; all partnership variables refer to the past six months; "serious" refers to the main effect of relationship type $(0=$ casual, $1=$ serious $)$; "bisexual" refers to the main effect of sexual orientation $(0=$ gay, $1=$ bisexual $)$; all estimates are adjusted for age, race/ethnicity, education, PrEP use, partnership HIV concordance, and whether or not the partnership was a repeat partnership. * $p<.05$

**

$p<.01$

**** $p<.001$ 
Table 5

Predictors of condomless sex acts in the subsample of self-identified bisexual men.

\begin{tabular}{lc}
\hline Predictor & $\begin{array}{c}\text { Condomless sex acts } \\
\text { IRR }(95 \% \text { CI })\end{array}$ \\
\hline Serious & $4.75(3.13,7.21)^{* * *}$ \\
Female & $1.41(0.77,2.58)$ \\
Alcohol & $2.29(1.50,3.50)^{* * *}$ \\
Marijuana & $3.84(2.52,5.85)^{* * *}$ \\
Serious x Female & $0.80(0.41,1.57)$ \\
Alcohol x Female & $0.69(0.33,1.41)$ \\
Marijuana x Female & $1.36(0.66,2.78)$ \\
\hline
\end{tabular}

Notes. IRR = incidence rate ratio; $\mathrm{CI}=$ confidence interval; all partnership variables refer to the past six months; "serious" refers to the main effect of relationship type $(0=$ casual, $1=$ serious $)$; "female" refers to the main effect of partner gender $(0=$ male, $1=$ female $)$; all estimates are adjusted for age, race/ethnicity, education, PrEP use, partnership HIV concordance, and whether or not the partnership was a repeat partnership.

* $p<.05$,

**

$p<.01$,

$* * *$

$p<.001$ 


\section{Table 6}

Predictors of substance use before sex in the subsample of self-identified bisexual men.

\begin{tabular}{lcc}
\hline Predictor & $\begin{array}{c}\text { Alcohol use before sex } \\
\text { OR }(95 \% \text { CI })\end{array}$ & $\begin{array}{c}\text { Marijuana use before sex } \\
\text { OR }(95 \% \text { CI })\end{array}$ \\
\hline Serious & $5.62(3.30,9.58)^{* * *}$ & $3.02(1.60,5.70)^{* * *}$ \\
Female & $3.16(1.7,5.88)^{* * *}$ & $3.37(1.62,7.02)^{* *}$ \\
Female x Serious & $0.37(0.15,0.89)^{*}$ & $0.76(0.26,2.22)$ \\
\hline
\end{tabular}

Notes. OR = odds ratio; $\mathrm{CI}=$ confidence interval; all partnership variables refer to the past six months; "serious" refers to the main effect of relationship type $(0=$ casual, $1=$ serious $)$; "female" refers to the main effect of partner gender $(0=$ male, $1=$ female $)$; all estimates are adjusted for age, race/ethnicity, education, PrEP use, partnership HIV concordance, and whether or not the partnership was a repeat partnership.

*

$p<.05$,

$* *$

$p<.01$,

**** $p<.001$ 